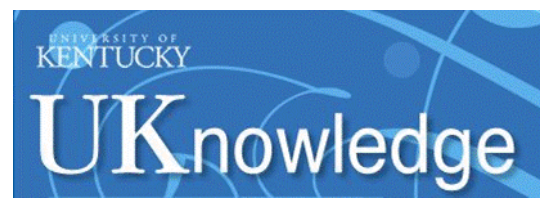

University of Kentucky

UKnowledge

2-18-2014

\title{
Secular Damping of Stellar Bars in Spinning Dark Matter Halos
}

\author{
Stacy Long \\ University of Kentucky, stacy.long@uky.edu \\ Isaac Shlosman \\ University of Kentucky, shlosman@pa.uky.edu \\ Clayton Heller \\ Georgia Southern University
}

Follow this and additional works at: https://uknowledge.uky.edu/physastron_facpub

Part of the Astrophysics and Astronomy Commons, and the Physics Commons

Right click to open a feedback form in a new tab to let us know how this document benefits you.

\section{Repository Citation}

Long, Stacy; Shlosman, Isaac; and Heller, Clayton, "Secular Damping of Stellar Bars in Spinning Dark Matter Halos" (2014). Physics and Astronomy Faculty Publications. 467.

https://uknowledge.uky.edu/physastron_facpub/467

This Article is brought to you for free and open access by the Physics and Astronomy at UKnowledge. It has been accepted for inclusion in Physics and Astronomy Faculty Publications by an authorized administrator of UKnowledge. For more information, please contact UKnowledge@lsv.uky.edu. 


\section{Secular Damping of Stellar Bars in Spinning Dark Matter Halos}

Digital Object Identifier (DOI)

https://doi.org/10.1088/2041-8205/783/1/L18

\section{Notes/Citation Information}

Published in The Astrophysical Journal Letters, v. 783, no. 1, L18, p. 1-5.

( 2 2014. The American Astronomical Society. All rights reserved. Printed in the U.S.A.

The copyright holder has granted the permission for posting the article here. 


\title{
SECULAR DAMPING OF STELLAR BARS IN SPINNING DARK MATTER HALOS
}

\author{
Stacy Long ${ }^{1}$, IsaAc Shlosman ${ }^{1,2}$, and Clayton Heller ${ }^{3}$ \\ ${ }^{1}$ Department of Physics and Astronomy, University of Kentucky, Lexington, KY 40506-0055, USA \\ 2 Theoretical Astrophysics, Department of Earth and Space Science, Osaka University, Osaka 560-0043, Japan \\ ${ }^{3}$ Department of Physics, Georgia Southern University, Statesboro, GA 30460, USA \\ Received 2013 December 11; accepted 2014 February 2; published 2014 February 18
}

\begin{abstract}
We demonstrate using numerical simulations of isolated galaxies that growth of stellar bars in spinning dark matter halos is heavily suppressed in the secular phase of evolution. In a representative set of models, we show that for values of the cosmological spin parameter $\lambda \gtrsim 0.03$, bar growth (in strength and size) becomes increasingly quenched. Furthermore, the slowdown of the bar pattern speed weakens considerably with increasing $\lambda$ until it ceases completely. The terminal structure of the bars is affected as well, including extent and shape of their boxy/ peanut bulges. The essence of this effect lies in the modified angular momentum exchange between the disk and the halo facilitated by the bar. For the first time we have demonstrated that a dark matter halo can emit and not purely absorb angular momentum. Although the halo as a whole is not found to emit, the net transfer of angular momentum from the disk to the halo is significantly reduced or completely eliminated. The paradigm shift implies that the accepted view that disks serve as sources of angular momentum and halos serve as sinks must be revised. Halos with $\lambda \gtrsim 0.03$ are expected to form a substantial fraction, based on the lognormal distribution of $\lambda$. The dependence of secular bar evolution on halo spin, therefore, implies profound corollaries for the cosmological evolution of galactic disks.
\end{abstract}

Key words: dark matter - galaxies: evolution - galaxies: formation - galaxies: halos - galaxies: interactions galaxies: kinematics and dynamics

Online-only material: color figures

\section{INTRODUCTION}

Redistribution of angular momentum in astrophysical systems is a major driver of their dynamical and secular evolution. Galactic bars facilitate this process by means of gravitational torques, triggered internally (spontaneously) or externally (interactively). Important aspects of stellar bar evolution are still being debated - their origin and evolutionary changes in morphology, growth, and decay, are not entirely clear. Theoretical studies of angular momentum redistribution in disk-halo systems have been limited almost exclusively to nonrotating halos, following pioneering works on linear perturbation theory by Lynden-Bell (1962), Lynden-Bell \& Kalnajs (1972), Tremaine $\&$ Weinberg (1984), and Weinberg (1985), which underscored the dominant role of orbital resonances. Numerical simulations have confirmed the angular momentum flow away from disks embedded in axisymmetric (e.g., Sellwood 1980; Debattista \& Sellwood 1998, 2000; Tremaine \& Ostriker 1999; Villa-Vargas et al. 2009, 2010; review by Shlosman 2013) and triaxial (e.g., El-Zant \& Shlosman 2002; El-Zant et al. 2003; Berentzen et al. 2006; Berentzen \& Shlosman 2006; Heller et al. 2007; Machado $\&$ Athanassoula 2010; Athanassoula et al. 2013) halos. Resonances have been confirmed to account for the lion's share of angular momentum transfer (e.g., Athanassoula 2002, 2003; Martinez-Valpuesta et al. 2006; Weinberg \& Katz 2007), In this paradigm, the halo serves as the pure sink and the disk as the net source of angular momentum.

However, realistic cosmological halos are expected to possess a net angular momentum, acquired during the maximum expansion epoch (e.g., Hoyle 1949; White 1978) and possibly during the subsequent evolution (Barnes \& Efstathiou 1987; but see Porciani et al. 2002). Simulations have quantified the distribution of spin values, $\lambda \equiv J_{\mathrm{h}} / \sqrt{2} M_{\mathrm{vir}} R_{\mathrm{vir}} v_{\mathrm{c}}$, for cosmological dark matter (DM) halos to follow a lognormal distribution, where $J_{\mathrm{h}}$ is the angular momentum, $M_{\mathrm{vir}}$ and $R_{\mathrm{vir}}$ the halo virial mass and radius, and $v_{c}$ the circular velocity at $R_{\mathrm{vir}}$, with the mean value $\lambda=0.035 \pm 0.005$ (e.g., Bullock et al. 2001). Spinning halos can increase the rate of the angular momentum absorption-an issue brought up by Weinberg (1985) but never fully addressed since. Only recently has it been confirmed numerically that the bar instability timescale is indeed shortened for $\lambda>0$ (Saha \& Naab 2013). But these models had been terminated immediately after the bar instability had reached its peak, and hence avoided completely the secular stage of bar evolution.

The $\lambda=0$ halos consist of two populations of DM particles, prograde and retrograde (with respect to disk spin). The amount of angular momentum in each of these populations can vary from zero for nearly radial orbits, to a maximal one for nearly circular orbits. (Both extremes are mentioned for pedagogical reasons only.) These extremes in angular momentum correspond to extremes in velocity anisotropy. Various degrees of velocity anisotropy in the halo lie in between and represent a rich variety of dynamical models. Stellar bars mediate the angular momentum transfer in such disk-halo systems with a broad range of efficiencies. The current paradigm of stellar bar evolution assumes an idealized version of a nonrotating DM halo which cannot account for the whole bounty of associated processes. We address these issues in a subsequent paper (in preparation)

In this Letter we demonstrate for the first time that secular growth of galactic bars in spinning DM halos is damped more strongly with increasing $\lambda$, and this effect is the result of a modified angular momentum transfer. Section 2 describes our numerical methods. Results are given in Section 3.

\section{NUMERICS AND INITIAL CONDITIONS}

We use the $N$-body part of the tree-particle-mesh smoothed particle hydrodynamics code GADGET- 3 originally described 
in Springel (2005). The units of mass and distance are taken as $10^{11} M_{\odot}$ and $1 \mathrm{kpc}$, respectively. We use $N_{\mathrm{h}}=10^{6}$ particles for the DM halo and $N_{\mathrm{d}}=2 \times 10^{5}$ for stars. Convergence models have been run with $N_{\mathrm{h}}=4 \times 10^{6}$ and $N_{\mathrm{d}}=4 \times 10^{5}$, in compliance with the Dubinski et al. (2009) study of discrete resonance interactions between the bar and halo orbits. The gravitational softening is $\epsilon_{\text {grav }}=50 \mathrm{pc}$ for stars and DM. To simplify the analysis we have ignored the stellar bulge. The opening angle $\theta$ of the tree code has been reduced from 0.5 used in cosmological simulations to 0.4 which increases the quality of the force calculations. Our models have been run for $10 \mathrm{Gyr}$ with an energy conservation of $0.08 \%$ and angular momentum conservation of $0.05 \%$ over this time.

To construct the initial conditions, we have used a novel method introduced by Rodionov \& Sotnikova (2006); see also Rodionov et al. (2009). We provide only minimal details for this method, which is elaborated elsewhere. It is based on the constrained evolution of a dynamical system. The basic steps include (1) constructing the model using prescribed positions of the particles with some (non-equilibrium) velocities, (2) allowing the particles to evolve for a short time which leads to modified positions and velocities, (3) returning the particles to the old positions with the new velocities, and (4) iterating on the previous steps until velocities converge to equilibrium values. This results in the near-equilibrium dynamical system which is then evolved.

The initial disk has been constructed as exponential, with the volume density given by

$$
\rho_{\mathrm{d}}(R, z)=\left(\frac{M_{\mathrm{d}}}{4 \pi h^{2} z_{0}}\right) \exp (-R / h) \operatorname{sech}^{2}\left(\frac{z}{z_{0}}\right),
$$

where $M_{\mathrm{d}}=6.3 \times 10^{10} M_{\odot}$ is the disk mass, $h=2.85 \mathrm{kpc}$ is its radial scalelength, and $z_{0}=0.6 \mathrm{kpc}$ is the scaleheight. $R$ and $z$ represent the cylindrical coordinates.

The halo density is given by Navarro et al. (1996, hereafter NFW):

$$
\rho_{\mathrm{h}}(r)=\frac{\rho_{\mathrm{s}} e^{-\left(r / r_{\mathrm{t}}\right)^{2}}}{\left[\left(r+r_{\mathrm{c}}\right) / r_{\mathrm{s}}\right]\left(1+r / r_{\mathrm{s}}\right)^{2}},
$$

where $\rho(r)$ is the DM density in spherical coordinates, $\rho_{\mathrm{s}}$ is the (fitting) density parameter, and $r_{\mathrm{s}}=9 \mathrm{kpc}$ is the characteristic radius, where the power law slope is (approximately) equal to -2 , and $r_{\mathrm{c}}$ is a central density core. We used the Gaussian cutoffs at $r_{\mathrm{t}}=86 \mathrm{kpc}$ for the halo and $R_{\mathrm{t}}=6 h \sim 17 \mathrm{kpc}$ for the disk models, respectively. The halo mass is $M_{\mathrm{h}}=6.3 \times 10^{11} M_{\odot}$, and halo-to-disk mass ratio within $R_{\mathrm{t}}$ is $\sim 2$. Other ratios have been explored as well. Oblate halos with various polar-to-equatorial axis ratios, $q=c / a$, have been analyzed, with $0.8 \lesssim q \lesssim 1$. Here, we limit our discussion to cuspy halos with $q \sim 1$, and a small core of $r_{\mathrm{c}}=1.4 \mathrm{kpc}$. Other profiles, such as the large core NFW and isothermal sphere density profiles, have been implemented as well, and resulted in qualitatively similar evolution. Dispersion velocity anisotropy, $\beta$, has been constrained initially to be mild using the novel method of Constrained Evolution discussed above. Velocities have been taken to be isotropic in the central region and the anisotropy increased to $\beta \sim 0.3$ outside the disk.

Disk radial dispersion velocities have been taken as $\sigma_{\mathrm{R}}(R)=$ $\sigma_{\mathrm{R}, 0} \exp (-R / 2 h)$ with $\sigma_{\mathrm{R}, 0}=143 \mathrm{~km} \mathrm{~s}^{-1}$. This results in $Q=1.5$ at $R \sim 2.42 h$, and increasing values toward the center and outer disk. Vertical velocity dispersions are $\sigma_{\mathrm{z}}(R)=$ $\sigma_{\mathrm{z}, 0} \exp (-R / 2 h)$, with $\sigma_{\mathrm{z}, 0}=98 \mathrm{~km} \mathrm{~s}^{-1}$.
To form spinning halos, we have flipped the angular momenta, $J_{z}$, of a prescribed fraction of DM particles which are on retrograde orbits with respect to the disk, by reversing their velocities, in line with Lynden-Bell's (1960) Maxwell demon. Only $\lambda \sim 0-0.09$ models are discussed here. The $\lambda<0$ cases are simpler due to a decreased fraction of prograde halo particles able to resonate with the bar/disk particles (e.g., Christodoulou et al. 1995). The implemented velocity reversals preserve the solution to the Boltzmann equation and do not alter the DM density profile or velocity magnitudes (e.g., Lynden-Bell 1960, 1962; Weinberg 1985). For spherical halos, the invariancy under velocity reversals is a direct corollary of the Jeans (1919) theorem (see also Binney \& Tremaine 2008). The most general distribution function for such systems is a sum of $f\left(E, J^{2}\right)$, where $E$ is the energy and $J$ is the value of the total angular momentum (i.e., $J^{2}$ ), and of an odd function of $J_{z}$, i.e., $g\left(E, J, J_{\mathrm{z}}\right)$ (Lynden-Bell 1960). If $g \neq 0$, the spherical system has a net rotation around this axis.

We left the disk parameters unchanged, while halo models have varied spin $\lambda$. The value of $\lambda$ has been added to the model name using the last two significant digits, e.g., P60 means $\lambda=0.060$ and "P" stands for prograde.

\section{RESULTS}

All models presented here have an identical mass distribution, both in DM and stars. Hence, any differences in the evolution must follow from the initial distribution of angular momentum in DM halos and its redistribution in the bar-disk-halo system. Figure 1 displays the evolution of the stellar bars through the amplitudes of the Fourier $m=2$ mode, $A_{2}$, and their pattern speeds, $\Omega_{\mathrm{b}}$, for $10 \mathrm{Gyr}$. This timescale is probably close to the maximum uninterrupted growth of galactic disks in the cosmological framework, and hence to the lifetime of the bars. The normalized (by the monopole term $A_{0}$ ) bar amplitude has been defined here as

$$
\frac{A_{2}}{A_{0}}=\frac{1}{A_{0}} \sum_{i=1}^{N_{\mathrm{d}}} m_{\mathrm{i}} e^{2 i \phi_{\mathrm{i}}},
$$

for $R \leqslant 14 \mathrm{kpc}$. The summation is performed over all disk particles with the mass $m=m_{\mathrm{i}}$ at angles $\phi_{\mathrm{i}}$ in the disk plane. $\Omega_{\mathrm{b}}$ is obtained from the phase angle $\phi=0.5 \tan ^{-1}\left[\operatorname{Im}\left(A_{2}\right) / \operatorname{Re}\left(A_{2}\right)\right]$ evolution with time. We divide the evolution into two phases: the dynamical phase, which consists of the initial bar instability and terminates with the vertical buckling instability of the bars and formation of boxy/peanut-shaped bulges (e.g., Combes et al. 1990; Pfenniger \& Friedli 1991; Raha et al. 1991; Patsis et al. 2002; Athanassoula 2005; Berentzen et al. 2007). Buckling weakens the bar but does not dissolve it (Martinez-Valpuesta \& Shlosman 2004). Repeated bucklings increase the size of the bulge (Martinez-Valpuesta \& Shlosman 2005; Martinez-Valpuesta et al. 2006). One buckling has been observed in the models presented here; following it, the bar enters the second phase, that of secular evolution.

The most striking development observed in models of Figure 1 during the secular phase is an increased damping of the bar amplitude and a slower or absent bar growth for $\lambda \gtrsim 0.03$. The P00 model $(\lambda=0)$ displays healthy growth after buckling. The P30 and P45 bars have a slower growth rate than the P00 bar, and do not recover their pre-buckling strength even after 10 Gyr. But models P60 and P90 show no growth in $A_{2}$ at all. The corresponding pattern speed evolution, $\Omega_{\mathrm{b}}(t)$, for 


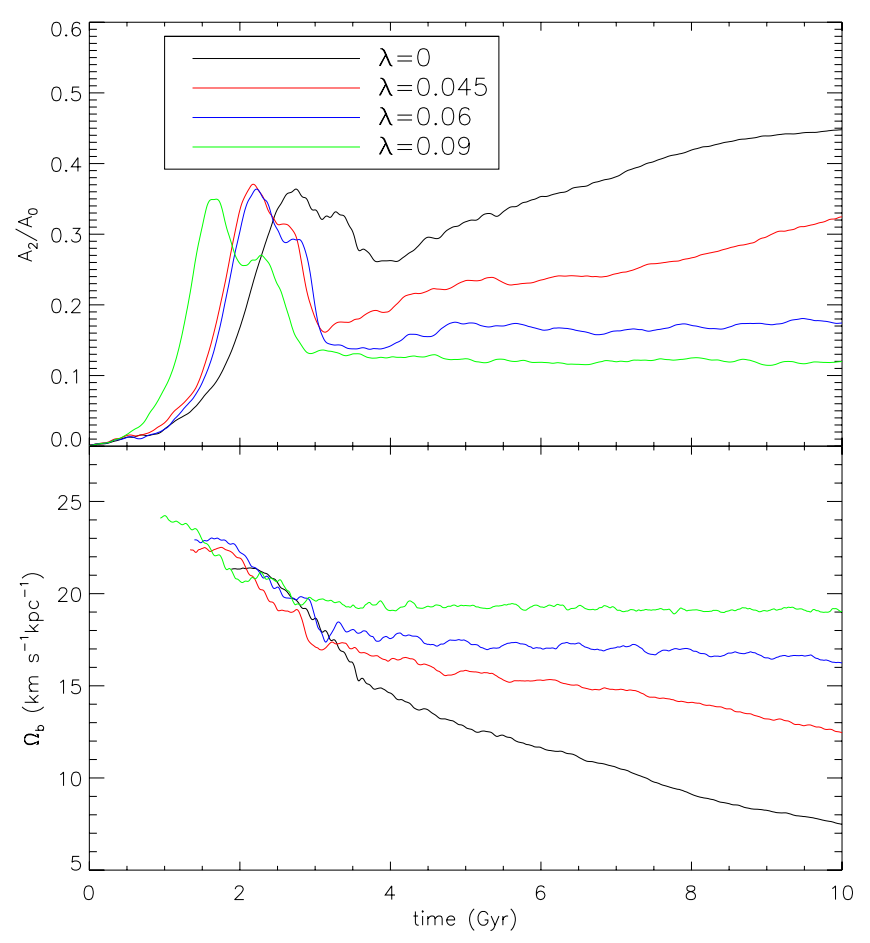

Figure 1. Upper: evolution of the bar amplitudes, $A_{2}$ (normalized by the monopole term $A_{0}$ ), for spherical NFW halos with $q=1$. Shown are P00, P45, P60, and P90 models. Lower: evolution of bar pattern speed, $\Omega_{b}$, in the above models.

(A color version of this figure is available in the online journal.)

these models differs substantially as well. The A90 bar displays a perfectly flat $\Omega_{\mathrm{b}}(t)$, and does not lose its angular momentum to the disk and/or the halo. This includes both the internal angular momentum (i.e., circulation) and the tumbling. A similar trend between the final $\Omega_{\mathrm{b}}$ and $\lambda$ can also be observed in Figure 7 of Debattista \& Sellwood (2000), although low resolution apparently prevented any conclusion of this sort.

Figure 2 compares the end products of the secular evolution of barred disks in models P00, P45, and P90. The differences appear to be profound. First, the bar size clearly anticorrelates with $\lambda$-this is a reflection of the inability of the bar potential to capture additional orbits and grow in length and mass. Second, the ansae (handles) feature is the strongest in the P00 bar, while it is smaller in size for P45 and completely absent in the P90 bar. Ansae have been associated with captured disk orbits librating around the bar (Martinez-Valpuesta 2006; Martinez-Valpuesta et al. 2006). This is another indication that the bar in high$\lambda$ models does not grow. Note that the surface density in the disk is clearly affected, as trapping of the disk orbits by the P00 bar creates low-density regions in the disk but not in P90. We analyzed the properties of the halo "ghost" bar (Holley-Bockelmann et al. 2005; Athanassoula 2007; Shlosman 2008) and found no growth there as well. The offset angle between the ghost and stellar bars remains near zero (within the error margin). Third, the face-on morphology of the P00 bar is that of a rectangular shape, while that of P90 is elliptical. Fourth, bulges that formed as a result of the buckling instability show the same anticorrelation trend in size $-\lambda$, as seen in edgeon (i.e., along the bar's minor axis) frames. Furthermore, they differ in shape as well: the P00 bulge has an X-shape, P45 is boxy/X-shaped, and P90 is boxy. Trapped three-dimensional orbits are responsible for the bulge shape (e.g., Patsis et al. 2002; Athanassoula 2005; Martinez-Valpuesta et al. 2006).
What is even more intriguing is the near or complete absence of secular braking in the P60 and P90 bars. Although the bars are weak, constancy of $\Omega_{\mathrm{b}}$ and $A_{2}$ over $6 \mathrm{Gyr}$ in $\mathrm{P} 90$ points to no angular momentum transfer away from the bar, or, alternatively, to an opposite flux from the halo which compensates for the loss of angular momentum by the bar. As we see below, it is the second possibility that takes place. While the P60 and P90 models exhibit extremes of this effect, it is visible at various levels in all models with $\lambda \gtrsim 0.02$.

While most of the angular momentum transfer away from the bar is due to resonances, we deal with this aspect of the problem elsewhere. However, we do quantify the rate of the overall angular momentum transfer between the disk and the halo, i.e., accounting for the resonant and non-resonant angular momentum redistribution. This is accomplished by dividing the disk and halo into nested cylindrical shells and constructing a two-dimensional map of the angular momentum change in each shell as a function of $R$ and $t$ (e.g., Villa-Vargas et al. 2009, 2010). Such a color-coded diagram is shown in Figure 3 for disk stars (lower frames), $\left\langle\dot{J}_{*}\right\rangle \equiv\left(\partial J_{*} / \partial t\right)_{\mathrm{R}}$, and for halo particles (upper frames $),\left\langle\dot{J}_{\mathrm{DM}}\right\rangle \equiv\left(\partial J_{\mathrm{DM}} / \partial t\right)_{\mathrm{R}}$, where the brackets indicate time-averaging.

The diagrams for P00 are the easiest to understand. The red (blue) colors correspond to the absorption (emission) of the angular momentum. The continuity of these colors for the P00 disk represents the emission and absorption of angular momentum by the disk prime resonances. For example, the dominant blue band drifting to larger $R$ with time is associated with the emission of angular momentum by the inner Lindblad resonance (ILR) and the additional blue band corresponds to the ultraharmonic resonance. The dominant red band follows the corotation resonance (CR) and the outer Lindblad resonance (OLR).

The number of DM particles on prograde orbits has steadily increased with $\lambda$, raising the possibility of resonant coupling between them and the bar orbits. This is supported by linear theory (Weinberg 1985 and references therein) and by numerical simulations (Saha \& Naab 2013). Indeed, we observe increased emission of angular momentum by the ILR and corresponding enhanced absorption by the halo. Halo particles are late to pick up the angular momentum from the bar (due to their higher velocity dispersion), but the exchange is visible already before buckling. Enhanced coupling between the orbits is the reason for the shorter timescale for bar instability.

The secular evolution of bars, however, proceeds under quite different conditions. The bar cannot be considered as a linear perturbation, and the halo orbits have already been heavily perturbed and some have been captured by the stellar bar. So, one expects the nearby halo orbits around the bar to be tightly correlated with the bar. The upper frames in Figure 3 display the rate of angular momentum flow in the DM halo. While the P00 halo appears to be completely dominated by the absorption of angular momentum at all major resonances (ILR, CR, OLR), P30 shows a quite different behavior and emits it at the ILR. The loss of angular momentum in this region of the DM halo is even more intense in P90. Already, at the buckling we can observe a weak blue band of emission in the P30 halo, alongside a strong absorption, instead of pure absorption in P00. Note that linear resonances shown by continuous curves appear to be a bad approximation to the actual nonlinear resonances given by the color bands because they are calculated under assumption of circular orbits. In the $\mathrm{P} 90$ halo, a strong emission is visible at the position of the disk ILR, which continues as a band 


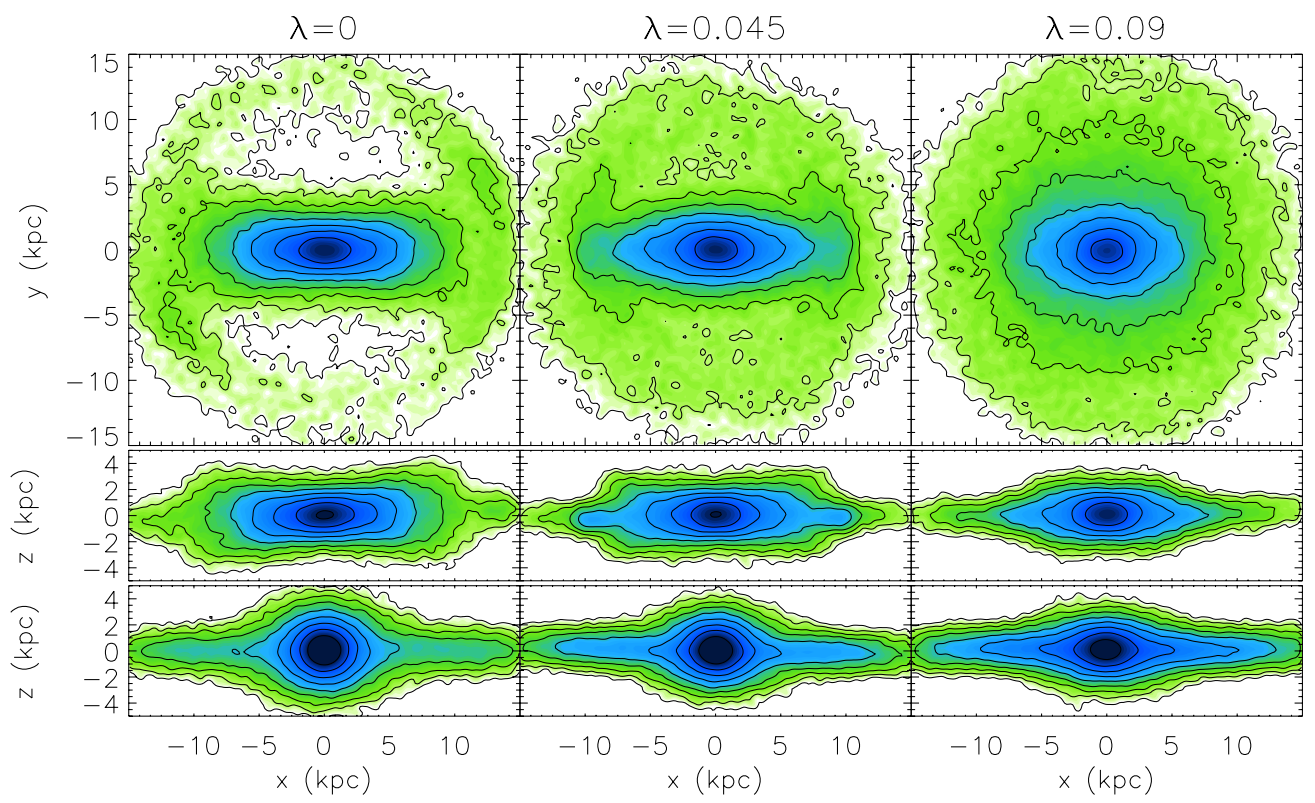

Figure 2. Disk-bar surface density contours (face-on, edge-on, and end-on) at $t=10 \mathrm{Gyr}$, for the NFW halos with $q=1$, P00 (left column), P45 (center), and P90 (right) models. Note the different bulge shapes: X-shaped for P00, boxy/X-shaped for P45, and boxy for P90, as well as decreasing strength of ansae with increasing $\lambda$ (see the text).

(A color version of this figure is available in the online journal.)

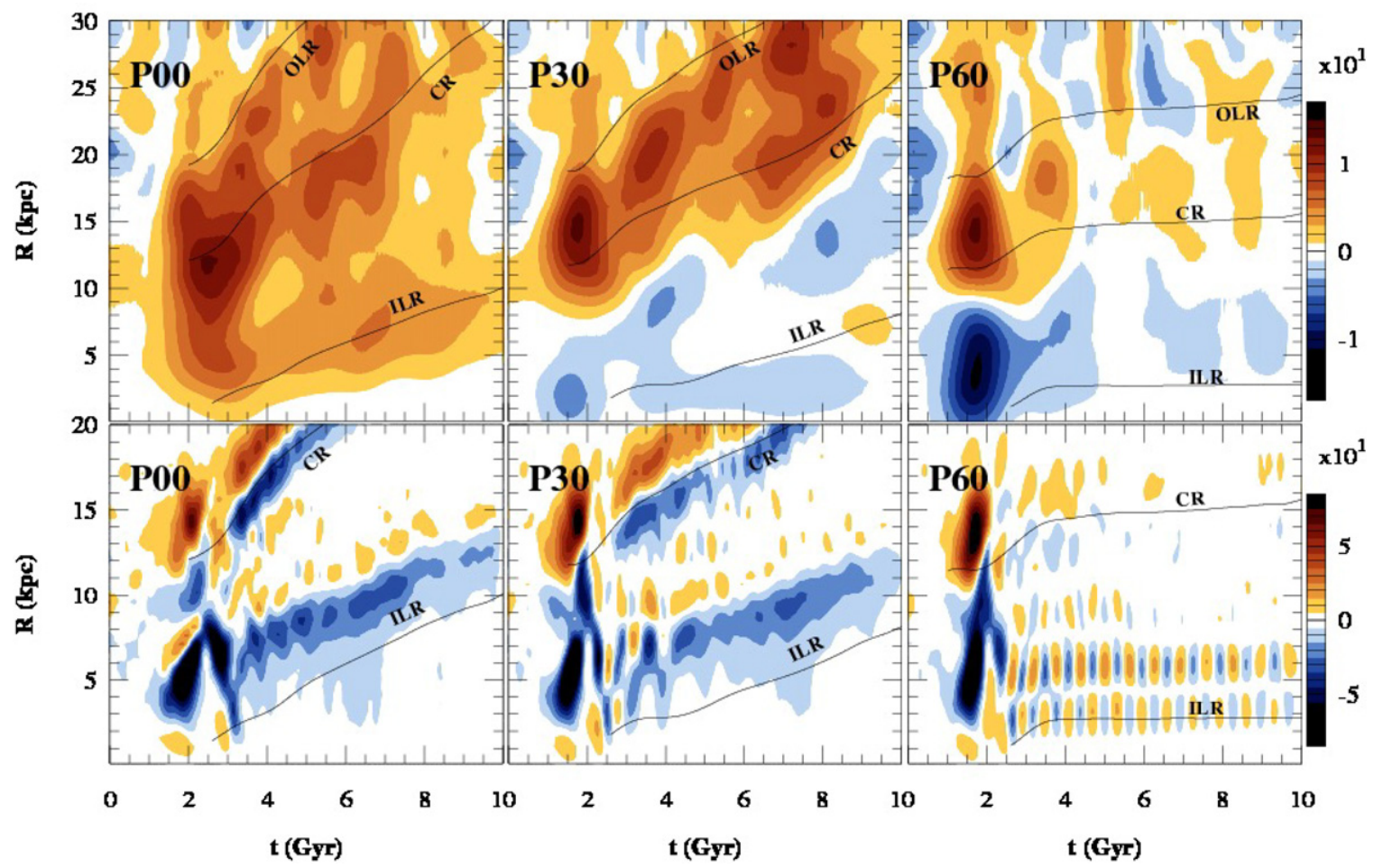

Figure 3. DM halos (upper frames): rate of angular momentum flow $j$ as a function of cylindrical radius and time for the P00 (left), P30 (middle), and P60 (right) models with $q=1 \mathrm{NFW}$ DM halos. The color palette corresponds to gain/loss rates (i.e., red/blue) using a logarithmic scale in color. The cylindrical shells have $\Delta R=1 \mathrm{kpc}$, extending to $z= \pm \infty$. Stellar disks (lower frames): same for (identical) disk models embedded in the P00 (left), P30 (middle), and P60 (right) halos, except $\Delta R=0.5 \mathrm{kpc}$, and $|\Delta z|=3 \mathrm{kpc}$. Positions of major disk resonances, ILR, CR, and OLR, have been marked.

(A color version of this figure is available in the online journal.)

alongside weakened absorption. So the absorption gradually weakens and moves out with increased $\lambda$, while the emission strengthens and spreads. The disk emission and absorption by major resonances also differs with changing $\lambda$-it gradually develops an intermittent behavior, especially at the ILR in P60, where the blue and red bands become intermittent. Such a cyclical behavior is not seen in the P00 disk, but becomes visible in the P30 disk and dominates the inner P60 disk. Hence, the spinning halo appears to emit and absorb angular momentum recurrently. The halo as a whole still absorbs the angular momentum from the disk in P30, while the net flux is zero for P90. 
This result is anticipated. The ability to pump angular momentum into a selected number of halo particles by means of a stellar bar is not without limits. As the angular momentum of the prograde population in the halo is increased, its ability to absorb angular momentum should saturate, and, under certain conditions, even be reversed. After buckling, the bar weakens substantially, as seen in Figure 1. At later stages, as the bar is expected to resume its growth, the near (disk) halo orbits can possess more angular momentum than the bar region which has been losing it for some time. For this prograde population, increase of $\lambda$ simply increases the initial angular momentum and the saturation comes earlier. What emerges as a fundamental property of a DM halo is the angular momentum and its distribution for the prograde population of orbits, irrespective of the value of $\lambda$.

Evolution of galactic bars is inseparable from the cosmological evolution of their host galaxies. We find that the secular growth of bars is significantly anticorrelated with the halo spin for $\lambda \gtrsim 0.03$. This means that majority of halos will adversely affect the bar strength, and, therefore, the angular momentum transfer and the bar braking. Beyond dynamical consequences, bars in spinning halos will be systematically smaller, which will make their detection at larger redshifts more difficult. This trend can be further strengthened because, during mergers, for a limited time period of $\sim 1-2 \mathrm{Gyr}, \lambda$ has been shown to increase (e.g., Hetznecker \& Burkert 2006; Romano-Diaz et al. 2007; Shlosman 2013). Weaker bars are known to possess star formation along the offset shocks, unlike strong bars, and are less efficient in moving the gas inward. Furthermore, damping bar amplitude has implications for disk morphology, stellar populations, and abundance gradients.

To summarize, we have investigated the dynamical and secular evolution of stellar bars in spinning DM halos. In a representative set of numerical models, we find that the angular momentum flow in the disk-halo system is substantially affected by the momentum distribution in the prograde population of DM particles, and is not limited to the momentum flux from the disk to halo. The associated bar pattern speed slowdown is minimized and ceases for larger $\lambda$. This means that the bar does not experience gravitational torques and its amplitude remains steady, while the angular momentum, both internal circulation and tumbling, is preserved. This trend becomes visible for $\lambda \gtrsim 0.02$ and dominates the bar evolution for halos with $\lambda \gtrsim 0.03$. Because of a lognormal distribution of $\lambda$ with a mean value of $0.035 \pm 0.005$, a substantial fraction of DM halos will be affected. We analyze the rate of angular momentum change by subdividing the disk-halo system into nested cylindrical shells, and show that the DM halo can both absorb and emit angular momentum, resulting in a reduction of the net transfer of angular momentum from the disk to the halo. The ability of the halo material to both emit and absorb angular momentum has important corollaries.

We are grateful to Sergey Rodionov for guidance with the iterative method to construct initial conditions, and to Ingo Berentzen, Jun-Hwan Choi, Emilio Romano-Diaz, Raphael Sadoun, and Jorge Villa-Vargas for help with numerical issues. We thank Volker Springel for providing us with the original version of GADGET-3. This work has been partially supported by grants from the NSF and the STScI (to I.S.). Simulations have been performed on the University of Kentucky DLX Cluster.

\section{REFERENCES}

Athanassoula, E. 2002, ApJL, 569, L83

Athanassoula, E. 2003, MNRAS, 341, 1179

Athanassoula, E. 2005, MNRAS, 358, 1477

Athanassoula, E. 2007, MNRAS, 377, 1569

Athanassoula, E., Machado, R. E. G., \& Rodionov, S. A. 2013, MNRAS, 429, 1949

Barnes, J., \& Efstathiou, G. 1987, ApJ, 319, 575

Berentzen, I., \& Shlosman, I. 2006, ApJ, 648, 807

Berentzen, I., Shlosman, I., \& Jogee, S. 2006, ApJ, 637, 582

Berentzen, I., Shlosman, I., Martinez-Valpuesta, I., \& Heller, C. 2007, ApJ, 666,189

Binney, J., \& Tremaine, S. 2008, Galactic Dynamics (Princeton, NJ: Princeton Univ. Press)

Bullock, J. S., Dekel, A., Kolatt, T. S., et al. 2001, ApJ, 555, 240

Christodoulou, D. M., Shlosman, I., \& Tohline, J. E. 1995, ApJ, 443, 551

Combes, F., Debbash, F., Friedli, D., \& Pfenniger, D. 1990, A\&A, 233, 82

Debattista, V., \& Sellwood, J. A. 1998, ApJ, 493, 5

Debattista, V., \& Sellwood, J. A. 2000, ApJ, 543, 704

Dubinski, J., Berentzen, I., \& Shlosman, I. 2009, ApJ, 697, 293

El-Zant, A., \& Shlosman, I. 2002, ApJ, 577, 626

El-Zant, A., Shlosman, I., Begelman, M. C., \& Frank, J. 2003, ApJ, 590, 641

Heller, C. H., Shlosman, I., \& Athanassoula, E. 2007, ApJ, 671, 226

Hetznecker, H., \& Burkert, A. 2006, MNRAS, 370, 1905

Holley-Bockelmann, K., Weinberg, M. D., \& Katz, N. 2005, MNRAS, 363,991

Hoyle, F. 1949, in Problems in Cosmical Aerodynamics, ed. J. M. Burgers \& H. C. van de Hulst (Dayton, OH: Central Air Documents Office), 19

Jeans, J. H. 1919, Problems of Cosmogony and Stellar Dynamics (Cambridge: Cambridge Univ. Press)

Lynden-Bell, D. 1960, MNRAS, 120, 204

Lynden-Bell, D. 1962, MNRAS, 123, 447

Lynden-Bell, D., \& Kalnajs, A. J. 1972, MNRAS, 157, 1

Machado, R. E. G., \& Athanassoula, E. 2010, MNRAS, 406, 2386

Martinez-Valpuesta, I. 2006, PhD thesis, Univ. Hertfordshire

Martinez-Valpuesta, I., \& Shlosman, I. 2004, ApJL, 613, L105

Martinez-Valpuesta, I., \& Shlosman, I. 2005, in AIP Conf. Proc. 783, The Evolution of Starbursts, ed. S. Hüttemeister et al. (Melville, NY: AIP), 189 Martinez-Valpuesta, I., Shlosman, I., \& Heller, C. 2006, ApJ, 637, 214

Navarro, J. F., Frenk, C. S., \& White, S. D. M. 1996, ApJ, 462, 563 (NFW)

Patsis, P. A., Skokos, Ch., \& Athanassoula, E. 2002, MNRAS, 337, 578

Pfenniger, D., \& Friedli, D. 1991, A\&A, 252, 75

Porciani, C., Dekel, A., \& Hoffman, Y. 2002, MNRAS, 332, 325

Raha, N., Sellwood, J. A., James, R. A., \& Kahn, F. D. 1991, Natur, 352,411

Romano-Diaz, E., Hoffman, Y., Heller, C., et al. 2007, ApJ, 657, 56

Rodionov, S. A., Athanassoula, E., \& Sotnikova, N. Ya. 2009, MNRAS, 392, 904

Rodionov, S. A., \& Sotnikova, N. Ya. 2006, ARep, 50, 983

Saha, K., \& Naab, T. 2013, MNRAS, 434, 1287

Sellwood, J. A. 1980, A\&A, 89, 296

Shlosman, I. 2008, in ASP Conf. Ser. 390, Pathways Through an Eclectic Universe, ed. J. H. Knapen, T. J. Mahoney, \& A. Vazdekis (San Francisco, CA: ASP), 440

Shlosman, I. 2013, in Secular Evolution of Galaxies, ed. J. Falcon-Barroso \& J. H. Knapen (Cambridge: Cambridge Univ. Press), 555 Springel, V. 2005, MNRAS, 364, 1101

Tremaine, S., \& Ostriker, J. P. 1999, MNRAS, 306, 662

Tremaine, S., \& Weinberg, M. D. 1984, MNRAS, 209, 729

Villa-Vargas, J., Shlosman, I., \& Heller, C. H. 2009, ApJ, 707, 218

Villa-Vargas, J., Shlosman, I., \& Heller, C. H. 2010, ApJ, 719, 1470

Weinberg, M. D. 1985, MNRAS, 213, 451

Weinberg, M. D., \& Katz, N. 2007, MNRAS, 375, 460

White, S. D. M. 1978, MNRAS, 184, 185 